\title{
K - Kontenrahmen - nicht einer wie der andere
}

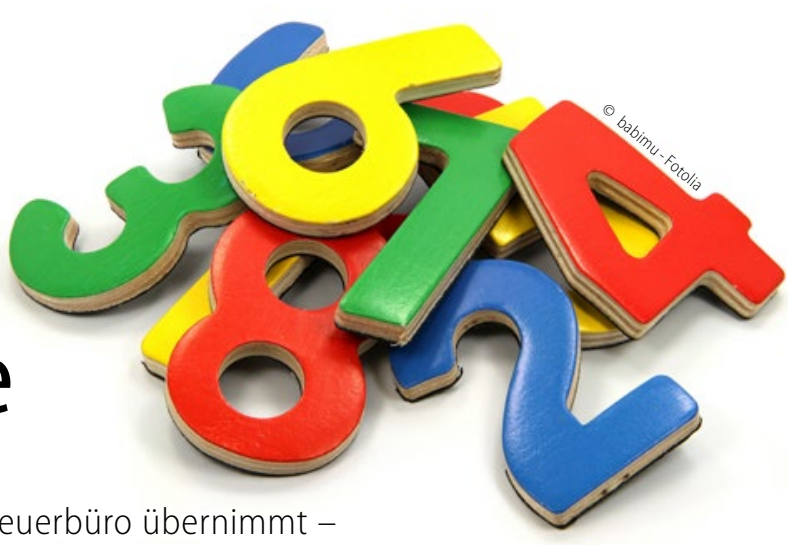

Egal, ob die Buchhaltung selbst erstellt wird oder diese Aufgabe ein Steuerbüro übernimmt die Begriffe "Konten" und "Kontenplan" tauchen unweigerlich auf. Vereinfachend ist zu sagen: Die Konten sind das Gitternetz, auf dem die Daten der betrieblichen Buchhaltung angeordnet werden, um anschließend wegen dieser Strukturierung eine Analyse zuzulassen, die einen Überblick über die Situation der Unternehmung erlaubt.

Kontenpläne wiederum entstehen aus Kontenrahmen. Der Kontenrahmen gibt eine Vielzahl von Konten vor, von denen das Unternehmen die relevanten verwendet, die also damit den individuellen Kontenplan des Unternehmens darstellen.

Als Standard haben sich die Kontenrahmen der DATEV etabliert. Wer sich auf der Seite der DATEV im Internet informiert, stellt fest, dass es eine Vielzahl von speziellen Kontenrahmen gibt, beispielsweise für Heime, soziale Einrichtungen oder verschiedene Auto-Marken. Diese sogenannten Branchen-Kontenrahmen verfügen in der Regel über eine geringere Anzahl von Konten, die speziell für die Anforderungen der Branche ausgewählt wurden und den Aufwand beim Steuerberater oder im Unternehmen vermindern sowie Betriebsvergleiche vereinfachen sollten.

Es ist aber ein erheblicher Aufwand, diese Kontenrahmen alle zu pflegen. Sicherlich nicht zuletzt deshalb hat die DATEV in den vergangenen Jahren regelmäßig Branchenkontenpläne abgekündigt und im Gegenzug die Eingliederung in die Standardkontenrahmen SKR 03 oder SKR 04 angeboten.

\section{Neues im Wirtschaftsjahr 2017}

So ergeht es nun zum Jahreswechsel 2016/2017 den Kontenrahmen SKR80 und SKR81 für Ärzte und Zahnärzte. Die Übernahme in das neue Wirtschaftsjahr 2017 ist laut DATEV im alten Format nicht mehr möglich, die Daten müssen in den SKR03 oder SKR04 transferiert werden. Dies gilt natürlich nur für die Anwender, die bisher auf den Branchenkontenrahmen gebucht haben. Wie dies technisch geht, ist letztlich eine Aufgabe, der sich die Steuerberater widmen müssen.

Für den Zahnarzt interessant ist jedoch die Frage, welchen Kontenplan das Steuerbüro zukünftig verwenden will. Für Selbstbucher ist es wichtig, frühzeitig ihren Steuerberater zu kontaktieren, um im folgenden Jahr im eigenen Buchhaltungsprogramm ebenfalls die Umstellung des Kontenrahmens rechtzeitig durchführen zu können. Gegebenenfalls ist hier noch Information bei den Software-Herstellern einzuholen.

Wo liegen die Unterschiede zwischen SKR03 und SKR04? Grundsätzlich sind beide Kontenrahmen geeignet, sie unterscheiden sich jedoch in der Art ihrer Gliederung. Der SKR04 ist nach dem Abschlussgliederungsprinzip aufgebaut, während sich der SKR03 nach den Prozessen eines Geschäftsbetriebes gliedert. Das heißt: Der SKR04 passt sich in seiner Struktur dem Aufbau einer Bilanz an, der SKR03 den Abläufen in der Praxis. Da SKR80 und 81 auf der Struktur des SKR03 aufbauten, ist anzunehmen, dass nach einer Umstellung im neuen SKR03 den Zahnärzten die Orientierung leichter fallen wird als bei einem Wechsel auf den SKR04. Wer also bei seinem Steuerbüro die Wahl hat, sollte sich für den SKR03 entscheiden, da dort die Kontonummern mit den bisherigen Kontonummern im Zahnärztekontenrahmen weitestgehend identisch sind.

\section{Verbuchung im sechsstelligen Branchenpaket möglich} Außerdem ist für beide Kontenrahmen zukünftig auch die Verbuchung in einem sechsstelligen Branchenpaket möglich. Auch hier sollten vor allem die Selbstbucher mit dem Steuerbüro eventuellen Umstellungsbedarf abklären, damit die verwendete Software angepasst werden kann.

Die Anbieter von Buchhaltungs-Software für Freiberufler im Gesundheitswesen haben sich genau wie die Anbieter von Praxissoftware frühzeitig mit diesem branchenspezifischen Problem auseinandergesetzt und ihre Software inzwischen weitgehend auf die neuen Erfordernisse eingestellt.

Unser Tipp: Für fibu-doc-Anwender stellt die Firma FIBU-doc Praxismanagement $\mathrm{GmbH}$ eine Anleitung zur Verfügung.

Mehr Infos unter www.fibu-doc.de

Das in den Artikeln dargestellte Buchhaltungskonzept basiert auf der zahnarztspezifischen Buchhaltungssoftware fibu-doc und wird vom FVDZ unterstützt.

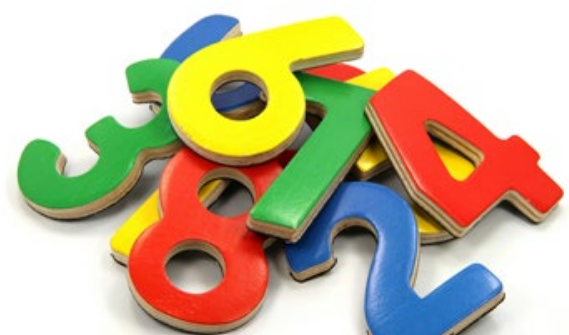

Barbara Mertens

www.fibu-doc.de 